\title{
Synthesis of Carbon NANODOT Particles from Natural Banana and its Metal Adsorption Studies
}

\author{
${ }^{1}$ O.T. Sangule, ${ }^{2}$ V.J.Sawant \\ Department of Chemistry, Smt. Kasturbai Walchand College, Sangli, M.S., India. \\ Affiliated to Shivaji University Kolhapur, Maharashtra. omsangule@ rediffmail.com
}

Summary - The carbon nanodot particles(CNDs) have focussed attention of scientists because of their versatality of sources for preparation and their multidisciplinary applications. Here ,we obtained CNDs by simple method using banana with $48 \%$ quantum yield. The synthesized carbon dots are characterized by using FTIR, Fluorescence spectroscopy. The metal $\mathrm{Fe}^{3+}$ shows adsorption on carbon nanodots. This property can be used for water remediation and purification purpose. Natural banana fruit was washed, cut into pieces and dried at $105^{\circ} \mathrm{c}$ for 24 hours. It was then carbonized at $400^{\circ} \mathrm{c}$ for 1.5 hours. Its carbon particles were activated in furnace at $600^{\circ} \mathrm{c}$. FTIR Spectra shows presence of primary amine group, carbonyl group, aromatic conjugated carbon chain. Further, PL Spectra represents n to $\pi^{*}$ and $\pi$ to $\pi *$ transitions, which shows presence of free electron cloud of $\mathbf{n} \& \boldsymbol{\pi}$ electrons. XRD shows amorphous nature, while TEM shows 4 to $6 \mathrm{~nm}$ sizes for nanoparticles. Metal adsorption graph of $\mathrm{Fe}^{3+}$ metal shows about 3.35 gm/mole adsorption on carbon nanodots. Carbon nanodots particles can be synthesized easily by wet chemical method by using natural banana. Freundlich and Langmuir isotherms are verified for $\mathrm{Fe}^{3+}$ metal adsorption by carbon nanoparticles. Costwise, synthesis of these nanoparticles is cheaper and can be used to remove metal ion from water.

Key words - Carbon nanodot particles, Banana, Metal adsorption, Characterisation.

\section{INTRODUCTION}

Nature provides an almost limitless supply of sources that inspire scientists to develop new materials with novel applications with eco-friendly manner. Recently, much attention has been focused on preparing natural-productderived carbon dots (CNDs) because natural products are renewable and have good biocompatibility ${ }^{1,2}$. They also contain heteroatoms, which facilitates the fabrication of heteroatom-doped CNDs without the addition of an external heteroatom source. Above all, some natural products can be used to prepare CNDs, which are very green and simple, relative to traditional methods for the preparation of carbon dots from man-made carbon sources ${ }^{3}$. CNDs have shown tremendous potential in many fields, including biosensing, bioimaging, optoelectronics, and photocatalysis ${ }^{4,5,6}$. This paper focussed recent progress in the synthesis, properties, applications, challenges and future direction of research on CNDs. This nanomaterials are composed mostly of carbon, most commonly taking the form of a hollow spears, ellipsoids or spheres or cubes. Carbon dots are small carbon nanoparticles less than $10 \mathrm{~nm}$ in size with some form of surface passivation . Carbon dots with $48 \%$ quantum yield were synthesized by simple hydrothermal treatment using banana as carbon source. Carbon nanodots (CNDs) are recently discovered fluorescent carbon nanoparticles with typical sizes of $<10 \mathrm{~nm}$. The CNDs have been reported to have excellent photophysical and chemical characteristic. In general, the synthetic methods for C-dots include arcdischarge, electrochemical oxidation, laser ablation, hydrothermal synthesis, pyrolysis, microwave-assisted heating, plasma treatment, neutralization heating and sonication treatment ${ }^{7}$. All these synthetic methods allow to prepare moderately high-quality C-dots with relatively high PL performance and small size. However, some of these synthetic methods suffer from limitations such as timeconsuming treatment process, requirement of strong acids for catalysis, high energy consumption and complex equipment set-up. Recently, there is a considerable interest in developing labour, material and energy efficient synthetic methods for CNDs such as carbonization of naturally available bio resources preparation with low heating temperature and synthesis without external heating. In addition, the flexibility in modification and functionalization of the CNDs surface has opened many possibilities in the incorporation of hetero atoms such as nitrogen, sulphur, phosphorous and silane into CNDs framework to enhance the PL properties of CNDs. Literature serve reveals that CNDs can be prepared from various sources like gelatin, soy milk ${ }^{1,2}$.etc. Further it has 
been revealed from literature that CNDs can acts as photocatalyst and shows luminescent properties ${ }^{8,9,10}$. Ofcourse combination of nanotechnology with numerous disciplines of science have driven the search of new advanced in nanoscale materials as a particular interest for scientist ${ }^{7}$. Here in this research work, we have synthesized CNDs from Banana source. After physicochemical characterization, we have used these CNDs for metal adsorption studies in water remediation.

\section{EXPERIMENTAL ANALYSIS}

\section{Materials}

The Banana procured from market was washed three times with water and peeled to get pieces. Then these pieces dried The same is explained in following flow diagram. in oven at ambient air temperature. Double distilled water was obtained from Millipore system. All other chemicals required for metal adsorption studies like Fe nitrate salt, EDTA, EBT indicator were procured from Merck ltd.

\section{Synthesis}

Firstly, banana was washed with tap water, dried and cut into small pieces. Then dried it in oven at $105^{\circ} \mathrm{c}$ for 24 hours. Grinded and dried small pieces of banana were carbonized at temperature $400^{\circ} \mathrm{c}$ for 1.5 hours. Ash of banana formed was dispersed in water, it was stirred for 3 hours on stirrer in double distilled water. The precipitate formed was dried in oven and fluorescent carbon nano powder was formed ${ }^{3}$

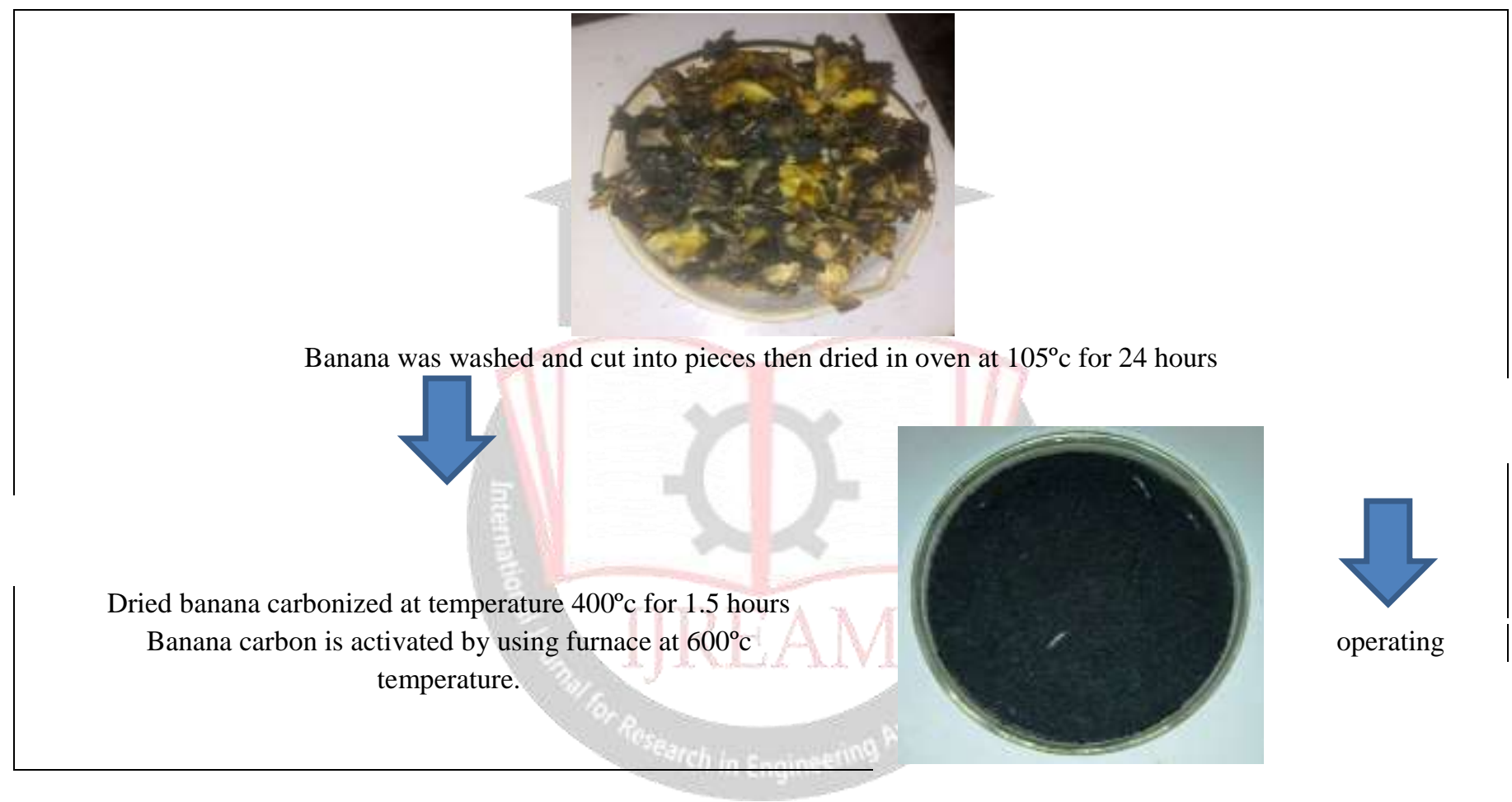

\section{Characterization}

Using PL, FTIR, XRD techniques and TEM microscopy the CNDs were characterized for morphology, size and composition. Riguku, Perkin elmer, Shimadzu instruments were used for the analysis.

\section{Metal adsorption isotherms for $\mathrm{Fe}$ (III)}

The Langmuir and Frendulich adsorption isotherms were elaborated for $\mathrm{Fe}$ (III) ions from nitrate media over CNDs using EDTA titration based metal adsorption estimations. 25 ppm solution of Fe(III) ions was studied for adsorption isotherms over CNDs to estimate capacity of metal adsorption of CNDs in water remediation.

\section{RESUlTS AND DISCUSSIONS}

\section{Physicochemical characterization}

The chemical composition, size, shape, and structure are important factors that determine the unique properties of
CNDs. Recently efforts have been made to explore the reliable and robust characterization tools for CNDs. UVVis and PL spectroscopy are used to measure the optical properties of CNDs. Here we have elaborated the morphology, size and composition of CNDs using FTIR, PL, XRD and TEM.

\section{FTIR Spectra}

FTIR Spectra (Fig1) is a widely used for characterization as tool to identify functionalities of the solid materials. In the case of IR analysis of CNDs samples, apart from the evaluation of hydroxyl $(-\mathrm{OH})$ and carbonyl $(\mathrm{C}=\mathrm{O})$ functional groups on the CNDs surface, FTIR is also able to examine the doping of heteroatoms into the CNDs framework. Important examples include the identification of the presence of amide/amine $\left(-\mathrm{CN} / \mathrm{NH}_{2}\right)$, alkyl sulfide (C-S), organosiloxane ( $\mathrm{Si}-\mathrm{O}-\mathrm{Si} / \mathrm{Si}-\mathrm{O}-\mathrm{C})$, phosphates $(\mathrm{P}=\mathrm{O}$ and $\mathrm{P}-\mathrm{O}-\mathrm{R}$ ) and boronic acid (B-O and $\mathrm{B}-\mathrm{N})$ moieties attached on the surface of CNDs if present on the basis of 
sources, providing evidence for introduction of nitrogen $(\mathrm{N})$, sulphur $(\mathrm{S})$, silicon (Si), phosphorus $(\mathrm{P})$ and boron $(\mathrm{B})$ hetero atoms into CNDs. The merits of this technique for the characterization of surface functionalization of CNDs are of low cost, simple, rapid and easy for sample preparation. However, the IR cannot give the fine structure information of CNDs and doping with metal heteroatoms such as aluminum $(\mathrm{Al})$, magnesium $(\mathrm{Mg})$, and nickel $(\mathrm{Ni})$ into CNDs cannot be revealed by FTIR. Here Fig. 1 explain the formation of CNDs from banana precursor, as fingerprint region of FTIR spectra shows conjugated aromatic and - COO linked chains of $\mathrm{C}$ dots. While functional group area provides information regarding some - $\mathrm{CO}$ and protein and peptide $-\mathrm{NH}_{2}$ groups from banana in CNDs, which reveals the composition of CNDs.

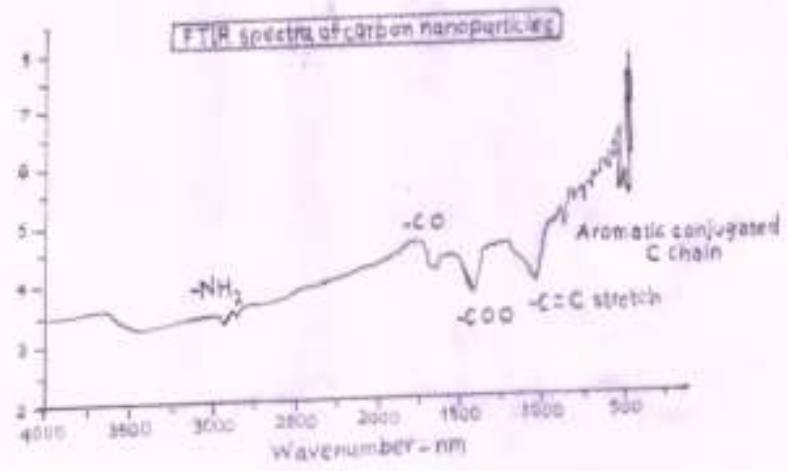

Figure 1

\section{PL Spectra}

PL spectroscopy are common and widely available spectroscopic techniques that have been used to measure the optical properties of CNDs. It represents $n \rightarrow \pi *$ and $\pi$ $\rightarrow \pi^{*}$ transitions, which shows presence of free electron cloud of $n \& \pi$ electrons as per fig. 2 .

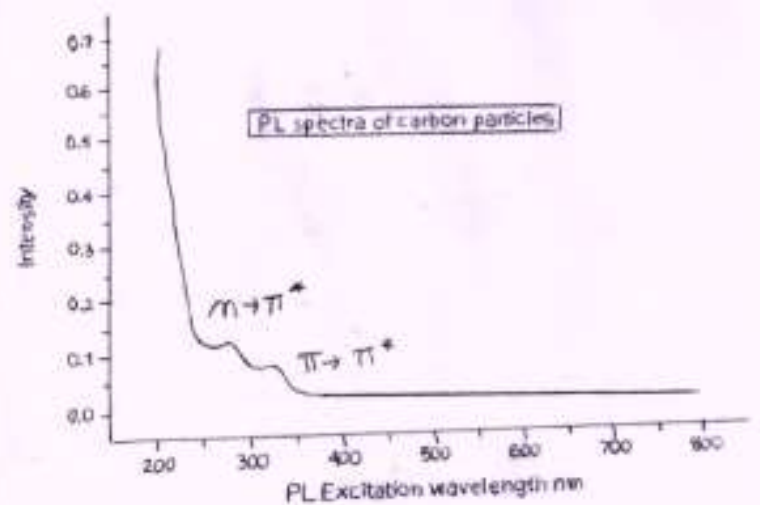

Figure 2

These PL spectra explain the presence of non-bonded and Pi electron clouds over surfaces of CNDs from their PL excitations. The peaks in spectra reveals the presence of strong non bonded and loose Pi clouds of electrons on CNDs. These properties are essential for metal and ion adsorptions.

\section{XRD spectra}

Fig. 3 shows XRD spectra of CNDs only one strong peak at theta proves presence of cubic [100] plane of these carbon nanomaterial particles. Hence it has been evidenced that the CNDs synthesized from banana contain cubic packing of carbon chains in 3D environment. As peak are blurred and broad the material is mostly amorphous in nature. From Scherer's equation size of nanomaterial was found as $5.7 \mathrm{~nm}$.

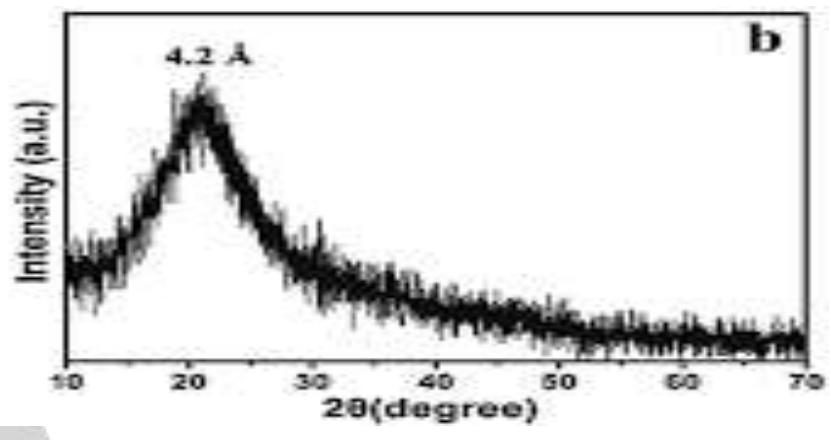

Figure 3: XRD spectra of CNDs

\section{TEM image}

As per figure 4 the CNDs synthesized from banana show mean particle diameter of $5 \mathrm{~nm}$. Nano particles exhibit spherical geometry, which contains aromatic and protein based aliphatic chains of carbon atoms with plenty of nonbonded and Pi electrons supported by PL and FTIR spectra. The TEM image morphology and size matches with XRD data.

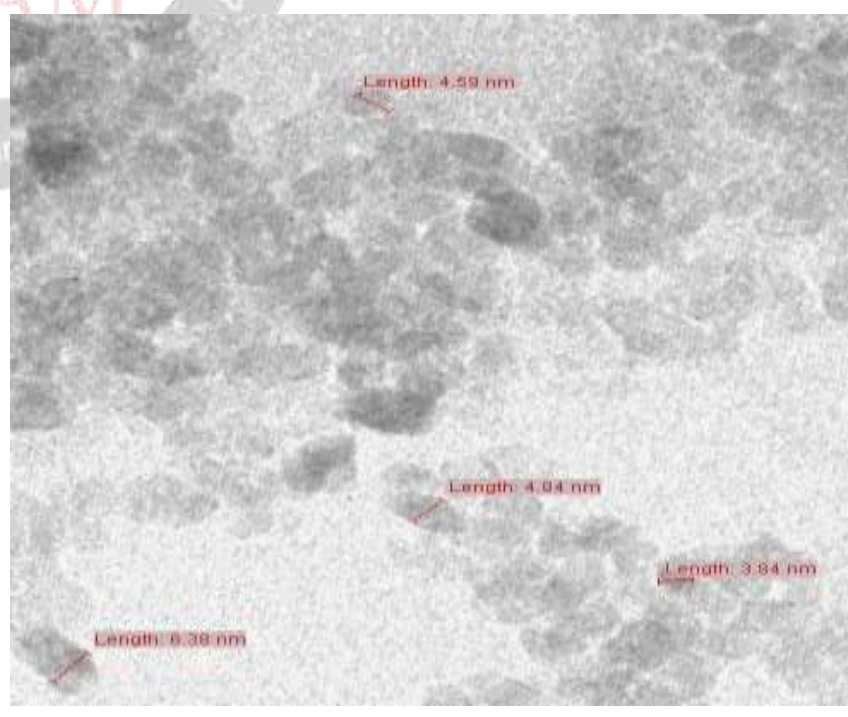

Figure 4: TEM image of CNDs from banana with mean particle diameter $5 \mathrm{~nm}$.

\section{Adsorption isotherms for $\mathrm{Fe}$ (III) adsorption}

The process of adsorption is usually studied by the plot known as adsorption isotherms. It is plotted by amounts of adsorbate (x) verses pressure at constant temperature. 
Different adsorption isotherms have been studied by freundlich and Langmuir.

Basic Adsorption Isotherm is in the process of adsorption. Adsorbate gets adsorbed on adsorbent.

$$
\text { Adsorbate + Adsorbent } \underset{\text { desorption }}{\stackrel{\text { Adsorption }}{\rightleftharpoons}} \text { Adsorption }
$$

$$
\mathrm{A}+\mathrm{B} \rightleftharpoons \mathrm{AB}
$$

(1)

In equation (1), A act as CNDs and B as ions. According to Le-Chatelier principle, the direction of equilibrium would shift in that direction where the stress can be relieved. In case of application of excess of pressure to the equilibrium system, the equilibrium will shift in the direction where the number of molecules decreases. As the number of molecules decreases in forward direction, increase in pressure will shift equilibrium in forward direction.

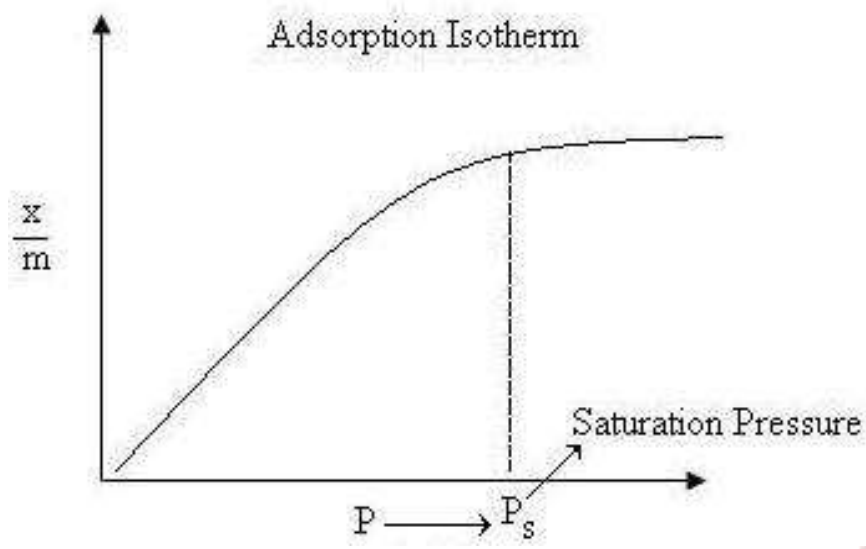

Graph 1: Adsorption Isotherm

Graph 1, explains that after saturation pressure Ps, adsorption does not occur anymore. This can be explained by the fact that there are limited numbers of vacancies on the surface of the adsorbent. At high pressure, a stage is reached when all the sites are occupied and further increase in pressure does not cause any difference in adsorption process. At high pressure, adsorption is independent of pressure.

In 1909, Freundlich gave an empirical expression representing the isothermal variation of adsorption of a quantity of gas adsorbed by unit mass of solid adsorbent with pressure. Equation (2) is known as Freundlich Adsorption Isotherm or Freundlich Adsorption equation or simply Freundlich Isotherm.

$$
\frac{x}{m}=k_{2} \sum^{\frac{1}{x}}
$$

Where $\mathrm{x}$ is the mass of the gas adsorbed on mass $\mathrm{m}$ of the adsorbent at pressure $\mathrm{p}$ and $\mathrm{k}, \mathrm{n}$ are constants whose values depend upon adsorbent and gas at particular temperature. Though Freundlich Isotherm correctly established the relationship of adsorption with pressure at lower values, it failed to predict value of adsorption at higher pressure. The same is shown in figure 5 .

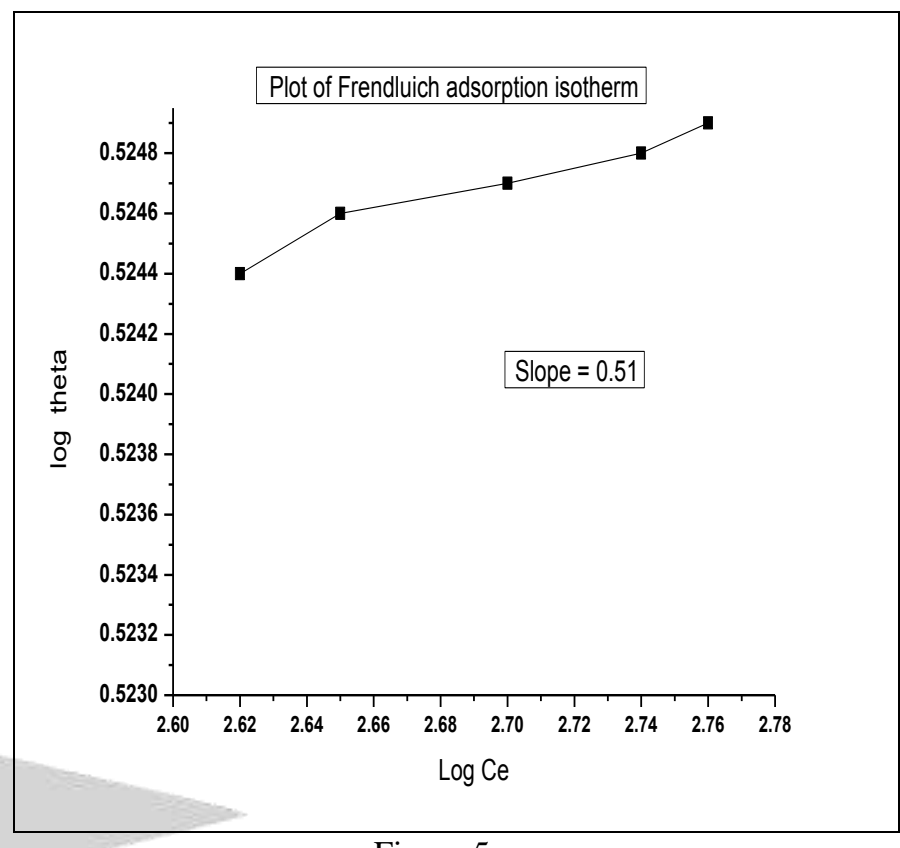

Figure 5

In 1916 Langmuir proposed another Adsorption Isotherm known as Langmuir Adsorption isotherm. One of the assumptions, on which this isotherm is based is that dynamic equilibrium exists between adsorbed gaseous molecules and the free gaseous molecules.

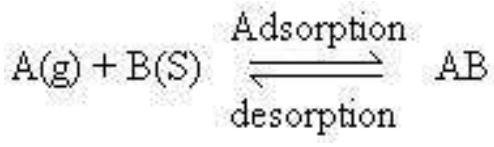

In equation (3), $\mathrm{A}(\mathrm{g})$ is unadsorbed gaseous molecule, while $\mathrm{B}(\mathrm{s})$ is unoccupied metal surface and $\mathrm{AB}$ is Adsorbed gaseous molecule.

In Langmuir Equation relationship between the number of active sites of the surface undergoing adsorption and pressure are depicted

$$
\theta=\frac{K P}{1+K P}
$$

In equation (4), $\theta$ means the number of sites of the surface covered with gaseous molecule, $\mathrm{P}$ represents pressure and $\mathrm{K}$ is the equilibrium constant for distribution of adsorbate between the surface and the gas phase. Limitation of Langmuir adsorption equation is that it is valid at low pressure only.

At lower pressure, $\mathrm{KP}$ is so small that factor $(1+\mathrm{KP})$ is almost negligible, hence Langmuir equation (4) reduces to

$$
\theta=\mathrm{KP}
$$

At high pressure, $\mathrm{KP}$ is so large, that factor $(1+\mathrm{KP})$ in denominator is nearly equal to KP. So Langmuir equation (4) reduces to 


$$
\theta=\frac{K P}{K P}=1
$$

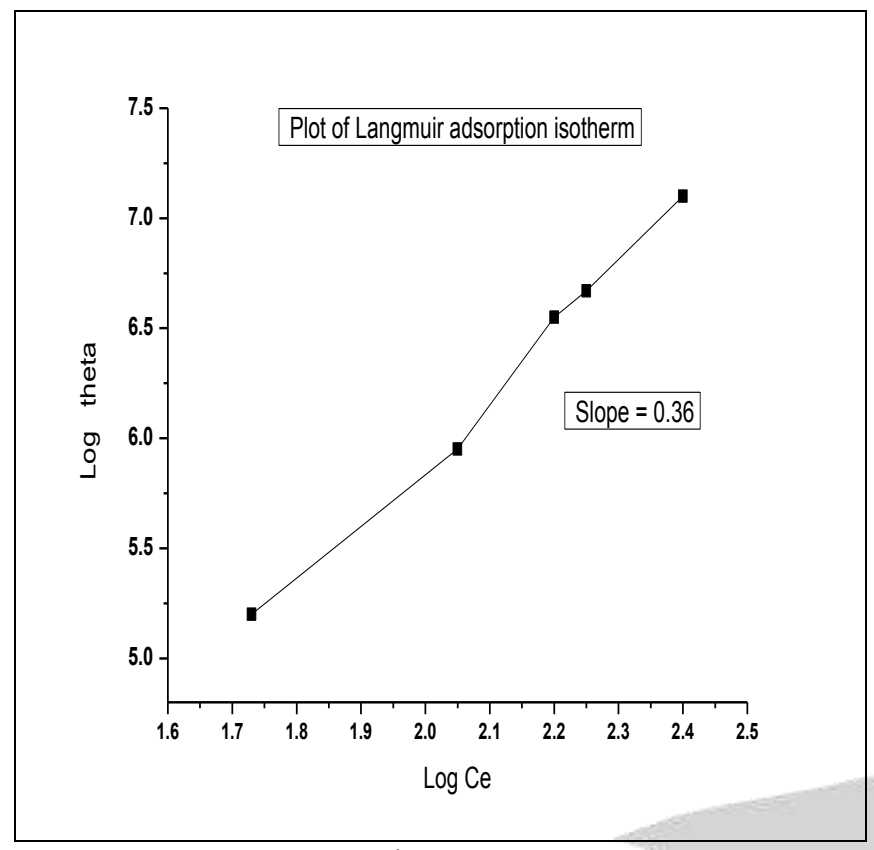

Figure 6

Here, Both the isotherms of $\mathrm{Fe}(\mathrm{III})$ adsorption on CNDs exhibited monolayer to multilayer adsorption probabilities based on parameters of water. At working $\mathrm{pH}=6.5-7.5$ the adsorption isotherms obtained with smooth linear curves. Fig..6 shows that $\mathrm{Fe}^{3+}$ adsorption on carbon nanodots is $3.35 \mathrm{gm} / \mathrm{mole}$.

\section{Conclusions}

Recently, combination of nanotechnology with other disciplines of science have driven the search of new advanced nanoscale materials as a particular interest for scientist. As an attempt, we followed green synthesis method for preparation of CNDs using banana. The banana is converted to CNDs by hydrothermal method like combustion. As synthesized material show the presence of conjugated aromatic and protein based chains in CNDs. The nanomaterial has shown good PL excitation. Spherical morphology have been confirmed on the basis of TEM image. Here very cheaper and handy method is used for production of carbon nanomaterials. Synthesized CNDs material is used for water purification purpose, particularly removal of metal ions[Fe(III)] and found pretty good results. Hence on the basis of metal adsorption studies of $\mathrm{Fe}$ (III), it proved that this nano material can be used for water remediation purpose in eco-friendly manner.

\section{ACKNOWLEDGEMENTS}

The authors acknowledge to faculty of Department of Chemistry, Smt. K. W. College, Sangli for providing laboratory facilities and to Department of Physics, Shivaji University, (Kolhapur) India for providing facility for characterisation of XRD and TEM.

\section{REFERENCES}

[1] Zhu C, Zhai J, Dong S., "Bifunctional fluorescent carbon nanodots: green synthesis via soy milk and application as metal-free electrocatalysts for oxygen reduction", Chem. Commun., 48(75):2012; 9367-9.

[2] Liang Q, Ma W, Shi Y, Li Z, Yang X., "Easy synthesis of highly fluorescent carbon quantum dots from gelatin and their luminescent properties and applications", Carbon, 60:2013; 421-8.

[3] Mewada A, Pandey S, Shinde S, Mishra N, Oza G, Thakur M, et al., "Green synthesis of biocompatible carbon dots using aqueous extract of Trapa bispinosa peel.", Mater Sci Eng C Mater Biol Appl, 33(5):2013; 2914-7.

[4] Wu N, Wang J, Tafen DN, Wang H, Zheng JG, Lewis JP, et al., "Shape-enhanced photocatalytic activity of single-crystalline anatase $\mathrm{TiO} 2$ (101) nanobelts.", J Am Chem Soc, 132(19):2010; 6679-85.

[5] Zhang H, Lv X, Li Y, Wang Y, Li J., "P25-graphene composite as a high performance photocatalyst", ACS Nano, 4(1): 2010; 380-6.

[6] Liu Y, Yu YX, Zhang WD., " Carbon quantum dotsdoped CdS microspheres with enhanced photocatalytic performance.", J Alloy Compd , 569(20):2013; 102-10.

[7] Li HT, Kang ZH, Liu Y, Lee ST., "Carbon nanodots: synthesis, properties and applications", J Mater Chem, 22,2012;:24230-53.

[8] Baker SN, Baker GA., "Luminescent carbon nanodots: emergent nanolights.", Angew Chem Int $E d$, 49,$2010 ;: 6726-44$.

[9] Bourlinos AB, Zbořil R, Petr J, Bakandritsos A, Krysmann M, Giannelis EP., "Luminescent surface quaternized carbon dots.”, Chem Mater, 24(1):2012; 6-8.

[10] Fang Y, Guo S, Li D, Zhu C, Ren W, Dong S, et al., "Easy synthesis and imaging applications of cross-linked green fluorescent hollow carbon nanoparticles", ACS Nano, 6(1):2012; 400-9. 